\title{
Ciberespaço e negociações de sentido: aspectos sociais da implementação de redes digitais de comunicação em instituições acadêmicas de saúde pública
}

\author{
Cyberspace and the negotiation of meaning: \\ implementing digital communications networks \\ in public health academic institutions
}

José Iturri 1

\footnotetext{
1 Centro de Documentação e Informação, Escola Nacional de Saúde Pública, Fundação Oswaldo Cruz. Av. Leopoldo Bulhões 1480, sala 310, Manguinhos, Rio de Janeiro, RJ 21041-210, Brasil. iturri@ensp.fiocruz.br iturri@centroin.com.br
}

\begin{abstract}
A bstract This articleanalyzes social aspects in the incorporation of new information and communications technologies in public health academic institutions. To demarcate the study of these processes and demonstrate the close relationship between their social and technical aspects, the study employs concepts pertaining to "intel lectual technologi es" and a "critical theory of technology". Theoretical and methodological el ements are identified to approach the implementation dynamics of el ectronic networks in public health institutions, through a discourse analysi s of their social actors and the various meanings they attribute to such dynamics Considering discourse as an expression of the relations created during these implementation dynamics, the study seeks a proposal for the ways by which these relations might influence the social and technical dimensions of digital networks.

Key words Public Health; Internet; Computer Communication Networks; Institutional Development; Technological Development

Resumo Analisam-se aspectos sociais da incorporação de novas tecnol ogias de informação e comuni cação em institui ções de ensi no e pesquisa da saúde pública. Usando el ementos da noção de tecnologias da inteligência e da teoria crítica da tecnol ogia para enquadrar o estudo dos processos de implementação de redes el etrôni cas de comuni cação (em especial Internet e intranets), aponta-se a ínti ma rel ação entre os aspectos sociais e técni cos desses processos. Traçam-se al guns el ementos teóri co-metodológi cos para aproxi mar-se das dinâmi cas de i mplementação de redes el etrônicas em insti tuições acadêmicas, medi ante a anál ise do discurso dos seus atores sociais e dos di versos senti dos que el es atri buem à implementação. Considerando o discurso como uma expressão das relações que os atores produzem durante a implementação, procura-se del inear uma aproximação às formas como essas rel ações influenciariam dimensões sociais e técnicas das redes di gitais.

Palavras-chave Saúde Pública; Internet; Redes de Comunicação de Computadores; Desenvolvimento Institucional; Desenvolvimento Tecnológico
\end{abstract}




\section{Introdução}

Nos últimos anos, tem-se formado um aparente descompasso entre os argumentos que advogam a favor da incorporação das tecnologias de redes digitais de informação (em especial Internet e intranets) nas atividades das instituições de ensino e pesquisa científica e o ritmo em que essa incorporação de fato acontece. A divergência entre as potenciais vantagens e o uso real da tecnologia de redes digitais pode ser constatada na literatura internacional (Harrison \& Stephen, 1996a; DeSieno, 1995; Eisenberg, 1997). Como se verá, existem alguns indícios recentes de que essa divergência possa estar presente também nas atividades de ensino e pesquisa de instituições acadêmicas de saúde pública no Brasil.

Este artigo se propõe a contribuir para dimensionar alguns aspectos das dinâmicas sociais que influenciam a incorporação de redes digitais em instituições de ensino e pesquisa científica. Pretende-se que tal dimensionamento seja de utilidade para a discussão do tema no campo da saúde pública.

A quase totalidade da literatura descreve as redes digitais de informação/comunicação como ferramentas poderosas para o trabal ho científico, com possibilidade de transformar completa e favoravelmente as estruturas e dinâmicas da prática acadêmica (Demo, 1997; Educom, 1996; Educom, 1995; Roszack, 1994). Essa visão progressiva e otimista parece estar baseada numa inferência lógica: já que a ciência é uma atividade essencial mente comunicacional (visto que a sanção do caráter científico de uma produção só se dá depois da sua disseminação, aceitação e uso pela comunidade dos cientistas) e que requer informações como seu insumo básico, seria de esperar-se que um dispositivo de comunicação tão potente quanto a Internet fosse rapidamente introduzido e adotado no meio acadêmico.

No entanto, contra o pano de fundo otimista mencionado, diversas avaliações de experiências e tendências reais na introdução de redes nas atividades de ensino e pesquisa científica falam em um "tempo inesperadamente prolongado" para que essas tecnologias transformem o cotidiano da vida acadêmica (Harrison \& Stephen, 1996b:3). Não raramente essas avaliações mencionam "ceticismo" (DeSieno, 1995), "necessi dade de rever o entusiasmo inicial" por parte do mundo científico (Eisenberg, 1995:32) ou um balanço de franca "subutilização das potencialidades" (Schwimmer, 1996:566). Aqui, porém, éimportante considerar que essa situação não é homogênea e que existem diversas áreas científicas nas quais o uso da rede virou um elemento central para sua produção, como é o caso de algumas ciências exatas e básicas (especialidades da Física, Matemática, Química, e Genética Molecular, por exemplo).

Alguns dos motivos para essa heterogênea, lenta e 'desconfiada' incorporação podem ser observados a partir da introduç̧ão de um artigo que faz um levantamento da utilização da Internet no campo da antropologia (Schwimmer, 1996). O autor, falando da potencialidade dessa tecnologia, afirma que ela "promete, ou talvez ameaça, transformar o caráter do trabal ho acadêmico" (Schwimmer, 1996:561). Isso é importante, pois apresenta a possibilidade de existir no mesmo meio - a academia - mais de uma interpretação e posicionamento diante de um dispositivo técnico. A rede poderia, então, não ser apenas uma ferramenta altamente eficaz. Segundo a perspectiva de alguns dos envolvidos na sua implementação, poderia ser "uma arma apontada contra nós". Um risco.

Por outro lado, a própria heterogeneidade dos processos de incorporação pode estar indicando que os ritmos de produção e estilos docentes (Educom, 1996), as histórias diferenciadas de disciplinas e instituições influenciam a adoção de um dispositivo como a Internet, que no discurso público é apresentado como um instrumento ideal para lidar com a informação, quer dizer, com aquilo que seria a base de toda e qualquer atividade científica. Talvez o instrumento não mexa apenas com informação, nem sequer no mundo acadêmico, que existe para gerar um tipo específico de informação.

Alguns dos artigos produzidos sobre o tema (DeSieno, 1995; Schwimmer 1996; Harrison \& Stephen 1996a, 1996b) permitem elaborar uma breve relação descritiva das dificuldades para a incorporação das redes digitais (em especial a Internet) no mundo acadêmico:

- Dificuldades para comprar, instalar e dar manutenção à base material básica e infra-estrutura da rede (computadores, programas, conexões); custo elevado dos investimentos iniciais para a instalação de redes. Estes são problemas da maior importância em países chamados subdesenvolvidos.

- Subutilização dos equipamentos já instalados (uso dos computadores essencialmente como máquinas de datilografar e não como elementos de rede).

- Resistência por parte dos integrantes da academia para aprender e desenvolver essa nova tecnologia. Tal resistência está relacionada à percepção de que essa tecnologia requer esforço e tempo desmedidos em comparação aos benefícios a serem obtidos. 
- Temores e fobias associados ao uso de computadores e redes (“Que, surpreendentemente, afetam os professores na mesma medida que qualquer outra pessoa", segundo Schwimmer (1996:566); temores por sua vez relacionados às mudanças sociais desencadeadas pelas novas tecnologias.

- Percepção de que a maior parte das aplicações dessa tecnologia estão desenhadas para atender a requisitos do mercado e não a necessidades específicas do ensino e pesquisa.

- Dificuldades para constituir processos de compartilhamento de informações entre colegas de instituição, com pesquisadores de outras instituições e com o público em geral.

- A estrutura etária dos corpos docentes de várias disciplinas científicas em que os departamentos e faculdades estão regidos. São pessoas "aci ma dos 50 anos que vêem pouca vantagem em fazer inovações estando já nos seusúltimos anos decarreira" (Schwimmer, 1996:566). - Percepção de que a redução de custos permitida pelas novas tecnologias equivaleria a uma redução de pessoal acadêmico.

Quase todas as referências até aqui mencionadas vêm de países chamados desenvolvidos, mas existem indícios no campo acadêmico da saúde coletiva brasileira que podem estar revelando um ritmo lento na incorporação da tecnologia de redes digitais. No primeiro semestre de 1997, num levantamento dos conteúdos das páginas web (home-pages) de instituições dessa área do saber - realizado pelo autor como pesquisador visitante da Escola $\mathrm{Na}$ cional de Saúde Pública/Fundação Oswaldo Cruz - comprovou-se que ainda era baixa a presença de informação científica e que nessas home-pages predominavam os dados institucionais e os anúncios de eventos; a referência a projetos científicos com elementos de colaboração em rede era praticamente nula e as publicações científicas em rede estavam restritas a duas revistas de caráter experimental, sem relação direta com os principais canais de publicação acadêmica. Esses indícios sobre as características da implementação de redes digitais podiam estar influenciados pelas limitações da infra-estrutura física (computadores, conexões etc.) e lógicas (programas, gerência) dessa tecnologia. Limitações que se inserem num contexto de mudança e restrição do financiamento público da ciência e tecnologia.

\section{Sobre tecnologias e academia}

Na procura de componentes teóricos que possam ajudar a dimensionar os elementos mencionados sobre a incorporação de redes digitais e explicar o aparente descompasso entre a promessa e a implementação, lançamos mão da noção de "tecnologias da inteligência" (Lévy 1993, 1997) e da "teoria crítica da tecnol ogia" (Feenberg, 1991, 1995). A primeira fornece as bases para começar a interpretar a relação entre as formas de representar/intercambiar conhecimento e as dinâmicas e organizações sociais que produzem esse conhecimento; por outro lado, é uma elaboração teórica centrada nas mudanças tecnológicas relacionadas à esfera do cognitivo, esfera que assumimos como importante na visão de mundo de atores sociais pertencentes à academia. Por sua parte, a teoria crítica da tecnologia, em primeiro lugar, permite uma referência mais ampla da tecnologia em geral na sociedade contemporânea e, em segundo, abre uma perspectiva distante do determinismo que desempenha um importante papel tanto nas análises científicas e filosóficas sobre tecnologia, quanto nas visões de mundo atualmente hegemônicas.

As tecnologias da inteligência (em especial a oralidade, a linguagem escrita e o meio eletrônico em rede) são consideradas simultaneamente suporte e parte integrante dos processos cognitivos. Elas não apenas expressam os pensamentos, como também influenciam as formas de pensar; aninham e nutrem de formas específicas para cada tecnologia os processos de conhecer. "Os processos intel ectuais não envolvem apenas a mente, colocam em jogo coisas e objetos técnicos compl exos defunção representativa e os automatismos operatórios que os acompanham." (Lévy, 1993:160)

A academia científica está fortemente influenciada pela sua relação com a comunicação escrita, contudo é importante lembrar que os cinco séculos de efeitos da impressão escrita "medem-se pel as formações sociais que ela possi bilitou enão pela tecnologia dos tipos móveis da imprensa" (Lyman, 1996:51). Da mesma forma, não se perceberá o impacto das novas tecnologias se centrarmos nossa atenção exclusivamente nos seus aspectos computacionais e de rede, sem situá-los no bojo das relações de força e associação que vão modulá-los.

Esta reflexão é feita num momento em que um grande conjunto de relações e valores da academia ligados à escrita e à publicação impressa entram em contato com uma nova tecnologia da inteligência: as redes digitais. Diversos autores assinalam que nenhuma tecnolo- 
gia de informação/ comunicação substitui completamente as anteriores (Lyman, 1996; Harrison \& Stephen, 1996b), portanto não estamos supondo que as redes do tipo Internet acabarão com a ampla e complexa acumulação social relacionada à escrita no caso da academia, porque, entre outras razões, essa acumulação está intimamente ligada aos interesses de uma indústria de publicação científica que tem grandes investimentos e está ainda adaptando-se ao novo meio (Kahin, 1996). Entretanto, é possível antecipar que essas duas ordens - a tradicional, ligada à escrita, e a que se prefigura, ligada às tecnologias de rede - vão entrar em um processo de adaptação conflituosa e, com base na literatura disponível, pode prever-se que nesse processo mostrar-se-ão as dinâmicas políticas e simbólicas que antes mencionamos.

A colocação de Lévy, segundo a qual: "A significação e o papel de uma configuração técnica em um momento dado não podem ser separados do projeto que move esta configuração, ou talvez dos projetos rivais que disputam-na epuxam-na em todos os senti dos. (...) nosso propósito consisteantes de mais nada em designar as tecnologias intelectuais como um terreno político fundamental, como lugar e questão de conflitos, de interpretações divergentes. Pois éao redor dos equi pamentos col eti vos da percepção, do pensamento e da comuni cação que se organiza em grande partea vida da cidade no cotidi ano eque se agenciam as subjetividades dos grupo" (Lévy, 1993:186-187), aponta para alguns elementos importantes para dimensionar o processo de implementação de redes eletrônicas em instituições. Mesmo que a história do uso de rede em meios acadêmicos brasileiros seja curta, esse uso já não está mais numa etapa inicial de introdução: é quase certo que a maior parte dos atores do processo já entraram em contato e têm diversos graus de familiaridade com as redes digitais; portanto, seus argumentos já estão baseados em um nível de manusei o e de tentativas (várias delas frustrantes) de uso e adaptação de redes el etrônicas, incluindo Internet. Nessas dinâmicas políticotécnicas, a abordagem das tecnologias da inteligência assinala a central idade da interpretação e da significação. É precisamente nessas duas últimas que centramos nossa atenção. As significações envolvidas num projeto técnico dizem tanto de "restrições econômicas (custos, patentes, situação do mercado, investimentos, estratégi as de desenvol vimento da firma) quanto sociais (qualificações, rel ações sociais implicadas à construção ou utilização da inovação), políticas (acessibilidade [dos insumos materiais], estado da legi slação [...], monopóli os do
Estado) ou culturais (relações com o público)" (Schlanger \& Stengers, 1988 apud Lévy, 1993: 188-189). Consideramos que é possível elaborar variáveis similares (sem dúvida com uma especificidade própria do campo) em firmas como as instituições acadêmicas de saúde pública.

É importante incluir nesse panorama as necessidades latentes da academia, que poderiam ser preenchidas pela inovação tecnológica, já que elas definirão muito do seu impacto (Lyman, 1996), e também as políticas de fomento e financiamento de ciência e tecnologia. Estas são produto da relação e negociações entre atores em um contexto político específico, que põe em contato seus interesses com mecanismos de poder de diversos níveis (federal, estadual, municipal), os quais definirão em parte o impulso que as novas tecnologias receberão. Essas políticas de ciência, por diversos motivos (relacionados a projetos políticos e econômicos), podem acabar favorecendo formas específicas de trabal ho e organização acadêmica baseadas em tecnologias de redes digitais. Ou não. Uma análise da implementação de redes eletrônicas de comunicação deveria ser feita a fim de acompanhar e estudar não só as políticas oficiais de órgãos como os Ministérios da Ciência e Tecnologia, de Saúde e de Educação, da Capes (Coordenação de Aperfeiçoamento de Pessoal de Nível Superior) e do $\mathrm{CNPq}$ (Conselho Nacional de Desenvolvimento Científico e Tecnológico), além do Conass (Conselho Nacional de Secretários da Saúde) e do Conasems (Consel ho Nacional de Secretários Municipais de Saúde), como também novos projetos ou órgãos de financiamento que concentrem recursos de importância.

Nesse panorama - e reconhecendo que existe já uma tradição científica que estuda o efeito dos meios de representar/intercambiar informação (especificamente a impressão em papel) sobre a história e organização da ciência -, a noção de tecnologias da inteligência de Lévy apresenta algumas vantagens por trabaIhar de forma especialmente aprofundada a relação dos meios antes usados (fala, escrita) com o mais recente (digital em rede) e por enfatizar a questão das forças e processos contemporâneos envolvidos na adoção do meio em rede.

Assim, com base principalmente em Lévy (1993), pode-se pensar que existiriam pelo menos duas formas de abordar o problema da implementação da Internet no campo acadêmico da saúde pública. Uma delas considera a rede como um problema essencialmente técnico e como um auxiliar dos processos de produção e 
divulgação de conhecimento e informação que a academia desenvolve há várias décadas; nesse sentido, a Internet não faria mais do que incrementar a velocidade e o 'colorido' desses processos. A outra abordagem assume, por um lado, a importância do caráter de negociação de interesses e das correlações sociais que dão apoio ou rejeitam determinadas propostas técnicas de implementação; por outro lado, sugere que a própria construção da presença da academia de saúde pública na rede e as modificações que ela induzirá nas instituições podem ser objeto da reflexão científica e, por esse caminho, transformar-se em um insumo estratégi co para a gestão da sua incorporação.

\section{Sobre tecnologias em geral}

A tentativa de caracterizar duas abordagens de implementação, que se pretende embasada em Lévy, guarda um paralelo com a noção de Feenberg de teorias "instrumentais" e "substantivas" sobre a tecnologia no mundo moderno (Feenberg, 1991; Feenberg \& Hannnay, 1995). A teoria instrumental está em geral associada à idéia de que a tecnologia é uma coisa boa; "a tecnologia não poderia ser 'má' ou controversa deforma alguma, pois tudo o que ela faz éajudar as pessoas a al cançar seus objeti vos preexistentes deforma mais eficiente" (Feenberg, 1991). Segundo a teoria substantiva, não é possível separar dessa forma os meios e os objetivos; a tecnologia não apenas serve para atingir tal ou qual fim; ela muda não só a forma de atingi-lo; a enorme importância da tecnologia nas nossas sociedades "muda o ambiente social; muda os obj eti vos a que nos propomos; muda o conteúdo de toda a nossa ação" (Feenberg, 1991). Noutras palavras, a tecnologia muda os fins. A teoria substantiva afirma que as mudanças que a tecnologia produz na sociedade não são apenas efeitos secundários; são mudanças profundas (por isso substantivas), que transformam a sociedade 'tecnologizando-a' e criando um ambiente tecnológico que permeia a sociedade toda. Ainda segundo esse autor, na abordagem substantiva "o ambiente tecnológi co é crescentementedesumanizado. Move-secada vez mais por imperati vos técni cos que emergem da estrutura do si stema de modo tal que os nossos fins têm cada vez menos a ver com esse ambi ente e têm cada vez menos lugar nele" (Feenberg, 1991). A tecnologia então acaba sendo uma coisa má.

Feenberg considera que essas duas abordagens teóricas são deterministas e reduzem drasticamente a capacidade de influência social deliberada sobre os processos tecnológi- cos. Propõe então uma terceira teoria que ele denomina teoria crítica da tecnologia, que resgata elementos das outras duas sob um outro enfoque. Situa-a na tradição da Escola de Frankfurt e embasa sua elaboração na leitura de Habermas, coincidindo com este autor na "necessidade de algum tipo de controle social da tecnologia" (Feenberg, 1991) que crie um processo no qual a racionalidade tenha um peso maior e não apenas responda aos acontecimentos. Sendo impossível fazer recuar a tecnologia ou amarrá-la, por causa do seu caráter intrínseco às sociedades modernas, ele afirma que se deve elaborar uma forma de trabalhar num "contexto fundamentalmente tecnológico e subvertê-lo onde el e precisar, transformá-lo onde precisar ser transformado, al terá-lo, reconstruí-lo" (Feenberg, 1991). Para isso, ele rejeita o determinismo tecnológico de modo tal que se possam fazer escol has sobre o papel da tecnologia, sem recuar, porém, até as posições da visão instrumental de que há apenas usos para a tecnologia. Afirma também que a tecnologia é humanamente controlável e propõe seu controle democrático; considera, porém, que o controle não é apenas do tipo "decisão sobreo uso de um determinado disposi tivo" (Feenberg, 1991), visto que esse não tem a forte carga cultural e social que as teorias substantivas identificam na tecnologia. Mas ele também toma distância da abordagem substantiva que considera que essas implicações culturais são prévias a qualquer decisão a respeito da tecnologia. Feenberg diz que o controle humano da tecnologia pode dar-se no nível do seu desenho e que: "Presumivel mente nesse nível, mudando o desenho em um sistema organizacional ou técnico, você poderia afetar os valores que eles carregam epoderia ser capaz decriar diferentes espaços nos quais sua ações têm significados fundamentalmentediferentes" (Feenberg, 1991).

A palavra-chave dessa última citação é "presumivelmente"; estamos diante de um campo com várias possibilidades abertas, nenhuma das quais tem seu sucesso garantido. E no que diz respeito à incorporação da Internet num campo do saber como a saúde pública, abremse problemas similares aos levantados por Feenberg para a tecnologia em geral.

A colocação de Harrison \& Stephen (1996b) ilumina bem o tipo de dinâmicas que dão lugar a estas possibilidades em aberto: "A tecnologia de redes por si só não determinará nenhuma mudança em particular no mundo acadêmico. As transformações que eventual mente atribuímos às novas tecnol ogias são melhor consi deradas como os produtos emergentes de negociações entre atores e grupos sociais ao longo do 
tempo, através das quais serão eventualmente respondidas questões como será usada a tecnologia, quem a usará equais grupos serão beneficiados (...) [as negoci ações estratégi cas desenvolvidas] incluem também as conseqüênci as de ações individuais, que, apesar do seu melhor esforço, os indi víduos não são capazes de controlar ou influenciar totalmente" (Harrison \& Stephen, 1996b).

Esses referenciais teóricos permitem começar a definir um olhar para analisar as questões da implementação aqui apresentada. A idéia central é lembrar que toda implementação de uma tecnologia tem um certo caráter de recriação desta e que todo projeto político (aqui incluídos os institucionais) ao mesmo tempo acompanha, usa e desvia a evolução de uma tecnologia (Lévy, 1993).

Desse ponto de vista, poder-se-ia então considerar que o aparente descompasso entre a promessa e a realidade da implementação da Internet seria uma expressão da situação atual dos processos, representados e negociados, de incorporação de uma tecnologia, do tipo específico das chamadas tecnologias da inteligência (com dimensões cognitivas, políticas, simbólicas) em um campo do saber como a saúde pública. É por isso que, nessa abordagem, a eventual não-implementação ou uma implementação parcial ou malsucedida de redes digitais também são objetos de reflexão. Nesses casos, interessa, talvez até mais do que em casos mais bem resolvidos, aproximar-se das dinâmicas que barram uma ferramenta de trabaIho que supostamente deveriam estimular.

\section{Negociando e representando espaços no ciberespaço}

Das diversas facetas do processo de implementação de redes digitais em instituições de um campo de saber que seriam de interesse apontar (por exemplo, os aspectos mais cognitivos, como as modificações que a rede digital introduz nas formas de procurar informação científica; ou aspectos mais epistemológicos, como as mudanças em mecanismos de validação de conhecimento científico introduzidos pelo uso da rede; ou alterações nas dinâmicas de publicação e citação de trabalhos científicos), enfatizamos neste artigo as relações que se estabelecem entre atores da implementação e as formas mediante as quais essas rel ações influenciam o ritmo e amplitude da implementação. No contexto de instituições acadêmicas, será de interesse aproximar-se dos papéis e das relações de três tipos de atores que participam da implementação: o corpo de professores/ pesquisadores, os gestores institucionais e os corpos especializados responsáveis pelos aspectos técnicos da implementação. Para essa aproximação, parece de utilidade a abordagem das representações sociais no seu sentido de expressões da relação entre linguagem e práxis social, e não apenas na acepção de discurso.

A representação social de um ator "visa dominar o ambiente, compreen der e explicar seus fatos e idéias, agi r sobre e com os outros, situarnos diante deles, comuni car com eles" (Arruda, 1992:119). De que maneira as representações sociais (que à primei ra vista aparentemente seriam apenas falas) podem estar relacionadas à ação e ser úteis para compreender um fenômeno como uma implementação de uma rede digital, uma coisa prática? Isso acontece porque as representações, no seu sentido de interpretação, relacionam-se com as interações de indivíduos e grupos (não existe a possibilidade de alguém "representar" livre da influência do seu conviver social). No contexto inevitável dessas interações, as representações são, por um lado, fruto das relações entre os seres humanos (portanto, ação) e, por outro, influenciam nessas mesmas interações (sendo, portanto, de novo... ação). Assim, "o conhecimento estudado via representações sociais é sempre um 'conhecimento práti co'; é sempre uma forma comprometida e/ou negociada de interpre tar a realidade" (Spink, 1993:303). Na aproximação aqui revisada, as representações servem para se comprometer e para negociar, com outros indivíduos e grupos sociais, a respeito do evento social da implementação de redes.

Pode-se adicionar mais um aspecto das representações que será de utilidade para dimensionar o processo: as representações são abordadas tendo como base os conteúdos mais cognitivos das falas, mas elas não são apenas componentes cognitivos (imagens, conceitos, categorias, teorias); elas devem ser entendidas também valendo-se do seu contexto de produção (as “funções si mból icas e ideológi cas a que servem e das formas de comuni cação onde circulam"). Spink cita Jodelet: "as representações sociais devem ser estudadas articulando el ementos afetivos, mentais, sociais, integrando a cogni ção, a linguagem ea comuni cação às relações sociais que afetam as representações sociais eà realidade material, social eideativa sobrea qual elasintervêm" (Jodelet, 1989 apud Arruda, 1992:123).

Aqui se deve lembrar, porém, que as representações sociais não são faladas pelas pessoas, não são dadas em depoimentos ou entrevistas, são construídas pela análise do discurso falado, procurando-se, nesse discurso e no seu 
contexto social, a significação que elas têm para o depoente. Para compreender melhor a relação entre o discurso (a fala) de indivíduos que integram grupos que se definem como atores e o contexto no qual ele é produzido, lançase mão do trabalho de Bakhtin. Parece-nos que nele fica claro "o papel da língua, como realidade material específica da criação ideológica" e a relação entre as lutas de interesses na sociedade e "a comunicação corriqueira". Este autor considera a palavra como "fenômeno i deológico por excelência" já que ela pode preencher qualquer função ideológica (Bakhtin, 1992:3637). Na palavra, refletem-se e refratam as tensões dos diferentes grupos e classes da sociedade; "a organização hi erarquizada das relações sociais" exerce uma "influência poderosa" sobre toda a produção de linguagem (Bakhtin, 1992:43). É por isso que o signo, a palavra, torna-se arena onde se desenvolvem confrontos entre grupos sociais. O fato de os diferentes grupos sociais fazerem uso de uma mesma língua, tendo interesses contrapostos na sociedade, permite explicar por que "em todo si gno ideológico confrontam-se índices de val or contraditórios" (Bakhtin, 1992:46).

Sem perder de vista que se pode supor que no processo de implementação de redes digitais os atores estabelecerão fortes relações de aliança e aproximação (devido às próprias histórias e contextos institucionais, origens sociais similares e interesses compartilhados pela academia), é útil considerar que as representações sobre a implementação da tecnologia de redes digitais estão atravessadas também pelas diversas significações os valores contraditórios que diferentes atores sociais Ihes outorgam; considera-se que essa tensão se reproduz nos discursos individuais.

Considerando, como antes vimos, o caráter das tecnologias intelectuais como um terreno político, lugar e questão de conflitos, de interpretações divergentes, toma-se a teoria das representações sociais como uma das aproximações que tem a vantagem de resgatar o caráter negociado e não necessariamente harmônico dessas relações durante a implementação.

Esta facilidade das representações sociais para lidar com relações e conflitos seria de interesse para dimensionar a implementação, em grande parte pelo contexto mais amplo no qual ela se insere; nesse sentido, considera-se provável que a restrição de financiamento público para ciência e tecnologia no Brasil repercuta e acirre a competição por recursos escassos no interior das instituições, assim como entre elas, e eleve as tensões sociais nesses espaços. Isso poderia definir situações de crises institucionais nas quais o papel (político e simbólico) atribuído às redes de informática mude muito. Nesse caso, o cenário mudará de forma mais ou menos imprevisível. A implementação de redes poderia ser simplesmente suspensa ou relegada a um segundo plano por falta de verba ou poderia ser colocada por algum ator institucional, ou por uma política nacional, como uma solução custo-benefício adequada para alguns problemas financeiros (reduzindo gastos em impressão, em meios de comunicação tradicionais, em publicações científicas etc.). Os cenários institucionais precisos não podem ser previstos com antecedência. Mas, para qualquer caso, existirão atores e interesses comprometidos com uma ou outra forma de encaminhar e resolver o assunto da implementação de redes digitais.

\section{Considerações finais}

A posição das instituições de ensino e pesquisa é particularmente interessante levando em consideração que sua atividade principal manuseio e produção de conhecimento é considerada como a grande favorecida pelo uso das tecnologias de redes digitais.

Mesmo sendo evidente que "ainda é cedo para fazer qualquer afirmação definitiva sobre as formas nas quais o uso das redes modi ficará o perfil e a estrutura da vida acadêmica" (Harrison \& Stephen, 1996b:7), parece claro que, qualquer que seja o rumo da implementação das redes de informação/comunicação, sua configuração específica dependerá principalmente da alteração das estruturas sociais relacionadas a disciplinas e formas de produção científica, da reestruturação das relações tradicionais entre professor e aluno e da desestabilização de antigas interdependências econômicas, legais e profissionais nos mecanismos de produção e disseminação de pesquisa acadêmica (Harrison \& Stephen, 1996b).

Nesse sentido, acreditamos que as abordagens e dimensões aqui resumidas possam servir, por um lado, de referências para conhecer melhor os determinantes sociais da tecnologia de redes digitais e do seu crescente papel no ensino e pesquisa de saúde pública e, por outro lado, para nos aproximar da intenção de “intervir no nível do desenho" dos arranjos tecnológicos, necessária para recuperar a possibilidade do seu "controle humano" e negar qualquer tipo de determinismo que deixa para as instituições apenas "a urgência de adaptar-se" à tecnologia (Feenberg, 1991; Feenberg \& Hannay, 1995; DeSieno, 1995). 


\section{Referências}

ARRUDA, A., 1992. Representações sociais: emergência e conflito na psicologia social. Anuário do Laboratório de Subjetividade e Política (UFF), 1:115-131.

DeSIENO, R., 1995. Netlaw: the faculty and digital technology. Educom Review, 30. Disponível na Internet em: http:// www.educom.edu/ web/ pubs/ review/ reviewArticles/30446.html. Arquivo consultado em nov. 1997.

EDUCOM., 1995. The promise and the peril. An interview with author Don Tapscott. By Educom Staff. Educom Review, 30. Disponível na Internet em: http:// www.educom.edu/ web/ pubs/ review/ reviewArticles/30532.html. Arquivo consultado em nov. 1997.

EDUCOM., 1996. Eli Noam on the future of the University. By Educom Staff. Educom Review, 31. Disponível na Internet em: http:// www.educom. edu/web/ pubs/review/ reviewArticles/31438.html. Arquivo consultado em nov. 1997.

EISENBERG, A., 1997. Disliking the internet. Scientific American, 276:32.

DEMO, P., 1997. Conhecimento Moderno: SobreÉtica e Intervenção do Conhecimento. Petrópolis: Vozes.

FEENBERG, A., 1991. Critical theory of technology. Conference at Monday, September 30, 1991, University Diamond Club, Vancouver, British Columbia, Canada. Disponível na Internet em: http:// bolt.lakeheadu.ca/ facedwww/Kerlin/tscp/ Feenberg.html. Arquivo consultado em nov. 1997.

FEENBERG, A. \& HANNAY, A., 1995. Technology and the Politics of Knowledge. Bloomington: Indiana University Press.

GUARESCHI, P. \&JOVCHELOVITCH, S., 1995. Textos em Representações Sociais. Petrópolis: Vozes.

HARRISON, T. \& STEPHEN, T., 1996a. Computer Networking and Scholarly Communication in the Twenty-First-Century University. New York: State University of New York Press.
HARRISON, T. \& STEPHEN, T., 1996b. Computer networking, communication, and scholarship. In: Computer Networking and Scholarly Communication in the Twenty-First-Century University. (T. Harrison \& T. Stephen, eds.), pp. 6-36, New York: State University of New York Press.

KAHIN, B., 1996. Institutional and policy issues in the development of the digital library. In: Computer Networking and Scholarly Communication in the Twenty-First-Century University. (T. Harrison \& T. Stephen, eds.), pp. 53-65, New York: State University of New York Press.

LÉVY, P., 1993. As Tecnol ogias da Intel igência. O Futuro do Pensamento na Era da Informática. Rio de Janeiro: Editora 34.

LÉVY, P., 1997. Collective Intelligence: Mankind's Emerging World in Cyberspace. New York/ London: Plenum Trade.

LYM AN, P., 1996. How is the medium the message?: Notes on the design of networked communication. In: Computer Networking and Scholarly Communication in the Twenty-First-Century University (T. Harrison \& T. Stephen, eds.), pp. 39-52, New York: State University of New York Press.

ROSCZACK, T., 1994. The Cult of Information. Berkeley: University of California Press.

SCHWIM MER, B., 1996. Anthropology on the internet: a review and evaluation of networked resources. Current Anthropology, 17:561-568.

SCIENTIFIC AMERICAN, 1997. The Internet: bringing order from chaos. Scientific American, 276:42.

SPINK, M. J., 1993. O conceito de representação social na abordagem psicossocial. Cadernos de SaúdePública, 9:300-308. 